\title{
DOS 'RECURSOS HUMANOS' À GESTÃO DO TRABALHO: UMA ANÁLISE DA LITERATURA SOBRE O TRABALHO NO SUS
}

\author{
FROM 'HUMAN RESOURCES'TO WORK MANAGEMENT: A REVIEW OF THE LITERATURE ON WORK IN \\ THE UNIFIED HEALTH SERVICES
}

\section{DE LOS 'RECURSOS HUMANOS' A LA GESTIÓN DEL TRABAJO: UN ANÁLISIS DE LA LITERATURA SOBRE \\ EL TRABAJO EN EL SUS}

Stela Maris Lopes Santini ${ }^{1}$

Elisabete de Fátima Polo de Almeida Nunes ${ }^{2}$

Brígida Gimenez Carvalho ${ }^{3}$

Francisco Eugênio Alves de Souza ${ }^{4}$

Resumo O estudo que deu origem a este artigo apresentou os resultados de revisão integrativa de literatura sobre a gestão do trabalho no Sistema Único de Saúde, tendo como objetivo analisar as diferentes contribuições científicas na área, as experiências e estratégias desenvolvidas pelos municípios. Utilizou-se para a coleta de dados as bases PubMed e SciELO em 2014. Após a aplicação dos critérios de inclusão e exclusão, analisaram-se 22 artigos. O maior número deles foi publicado no biênio 2010-2011 e referia-se a municípios de grande porte ou estados. Foram construídas duas categorias temáticas: concepções de gestão de trabalho e questões relacionadas à vida funcional do trabalhador, como provimento e garantia de direitos trabalhistas. Verificaram-se a evolução do termo recursos humanos para a concepção de gestão do trabalho; o processo de expansão dos empregos públicos na esfera municipal e as diversas formas de seleção adotadas; a desprecarização dos vínculos trabalhistas a partir dos anos 2000; dificuldades de atração e fixação de profissionais e a não consolidação do plano de carreira, cargos e salários como instrumento estratégico para a gestão do trabalho. Evidenciaram-se a complexidade do tema e a necessidade de constantes estudos pela sua importância para o Sistema Único de Saúde.

Palavras-chave gestão do trabalho; força de trabalho em saúde; gestão em saúde; recursos humanos em saúde; Sistema Único de Saúde.
Abstract The study that led to this article featured the results of an integrative review of literature on the management of work in the Unified Health System, and its purpose was to analyze the different scientific contributions, experiences, and strategies the municipalities developed in the area. The PubMed and SciELO databases were used for data collection in 2014. After applying the inclusion and exclusion criteria, 22 articles were analyzed. Most were published in the 2010-2011 biennium and referred to major cities or states. Two thematic categories were constructed: Work management concepts and issues related to the worker's functional life, such as provisioning and guaranteeing labor rights. It was noted that there has been evolution in the human resources term for the conception of work management; an expansion process in public employment at the municipal level and in the various forms of selection adopted; in employment bond stability from the 2000s, and that there are difficulties in attracting and securing professionals, as well as in the non-consolidation of the career, position and salary plan as a strategic tool for managing the work. The complexity of the issue and the need for constant studies were evident due to the importance of these factors for the Unified Health System.

Keywords work management; health workforce; health management; human resources in health; Unified Health System. 


\section{Introdução}

A gestão da força de trabalho é um fator determinante para a consolidação da universalização da cobertura e para a garantia da equidade das ações no Sistema Único de Saúde (SUS). As características do trabalho em saúde - um trabalho coletivo, multidisciplinar, relacional, que exige uma interação constante não só entre profissionais, mas também entre profissionais e usuários são uma das razões pelas quais os trabalhadores exercem papel estratégico para que o SUS alcance seus princípios e diretrizes. Tais características também reforçam a relevância e a complexidade da gestão do trabalho no SUS, exigindo mais atenção nas agendas políticas e técnicas e maior reconhecimento sobre a importância da força de trabalho em saúde (FTS).

Temas relacionados à gestão do trabalho, como planos de carreiras, cargos e salários (PCCSs), recrutamento e seleção, condições de trabalho, provimento de pessoal, remuneração, financiamento e fixação de profissionais estão sempre presentes, trazendo à tona o desafio diário que se apresenta aos gestores nessa área, principalmente aos municipais - porque nesses mais de 25 anos do SUS, o crescimento da oferta de empregos públicos se deu com a expansão do parque sanitário municipal (Campos, Machado e Girardi, 2009).

Tais questões demandam a necessidade de se estudar: como se processa e no que consiste a gestão do trabalho no SUS? Quais os determinantes de rotatividade/fixação da FTS? Quais as modalidades de seleção e admissão, e outras ações ou políticas desenvolvidas pelos municípios?

A revisão aqui apresentada teve por objetivo analisar o desenvolvimento e as estratégias utilizadas para a gestão do trabalho no SUS pelos municípios, por meio de uma revisão integrativa de literatura, pois conhecer tal processo poderá contribuir para o desenvolvimento de tecnologias de gestão em saúde adequadas para esse campo e ressignificar a gestão do trabalho como um importante eixo da estrutura organizacional dos serviços de saúde.

\section{Percurso metodológico}

Tratou-se de uma revisão integrativa de literatura elaborada com base na análise de artigos indexados na base de dados National Library of Medicine (PubMed) e Scientific Electronic Library Online (SciELO), cuja pergunta norteadora foi: "Como está sendo desenvolvida a gestão do trabalho no SUS e quais estratégias têm sido utilizadas pelos municípios?"

A fim de apoiar a busca de dados, utilizou-se a conceituação de gestão do trabalho apresentada por Ramos e colaboradores (2009): 
a gestão do trabalho no SUS envolve as questões relacionadas à vida funcional do trabalhador (ingresso, provimento, movimentação, enquadramento, desenvolvimento na carreira e garantia de direitos trabalhistas), as garantias de condições adequadas de trabalho, participação dos trabalhadores na formulação de políticas (Plano de Cargos, Carreiras e Salários) e na criação de espaços de negociação (Ramos et al., 2009, p. 44).

Para o levantamento de dados utilizou-se o cruzamento dos seguintes descritores/palavras-chave: sistema de saúde and Brasil and força de trabalho (health system and Brazil and labor force), ocupações em saúde (health occupations), recursos humanos (human resources), gestor de saúde (health manager or management of work), work regulation, Sistema Único de Saúde (Unified Health System or Single Health System), recursos humanos em saúde (health manpower), settlement strategies, reorganização de recursos humanos (turnover), personnel management, personnel downsizing, retention health professional, personnel selection, recursos humanos em saúde (health manpower).

Os critérios de inclusão foram: idioma (português, inglês e espanhol); sem limitação de data de publicação; artigos de fontes primárias, secundárias ou de revisão que respondessem ao objeto de estudo, isto é, o desenvolvimento da gestão do trabalho no SUS e as estratégias utilizadas pelos municípios para sua operacionalização; e disponibilidade (on-line com acesso gratuito). Como critérios de exclusão: livro ou capítulo de livro; dissertações, monografias, teses e documentos ministeriais.

A pesquisa foi realizada nos meses de abril e maio de 2014 (PubMed) e outubro de 2014 (SciELO). Para maior aprofundamento e detalhamento, os artigos que abordavam as questões de gestão do trabalho no SUS foram registrados em uma planilha contendo as seguintes informações: título, autores, revista e ano de publicação, descritores, objetivos, método, principais resultados e conclusão.

\section{Principais resultados}

\section{Caracterização dos artigos analisados}

No PubMed, foram inicialmente encontrados 99 artigos mediante leitura do título e resumo; no SciELO, 36. Do PubMed, excluíram-se 53 por não discutirem questões específicas sobre a gestão do trabalho no SUS; do SciELO, 16. Após leitura na íntegra dos 46 artigos restantes do PubMed, foram eleitos 12 que respondiam à questão da pesquisa; do SciELO, 26, sendo que dez encontravam-se em duplicidade com o PubMed. Dessa forma, foram utilizados 22 artigos nesta revisão (Tabela 1). 
Tabela 1

Caracterização dos artigos analisados conforme ano de publicação, periódico e local de estudo

\begin{tabular}{|c|c|c|}
\hline Caracterização & $\mathrm{N}^{\circ}$ & $\%$ \\
\hline \multicolumn{3}{|l|}{ Ano de publicação } \\
\hline 2001 & 01 & 4,5 \\
\hline 2002 & 02 & 9,1 \\
\hline 2004 & 01 & 4,5 \\
\hline 2007 & 01 & 4,5 \\
\hline 2008 & 02 & 9,1 \\
\hline 2009 & 02 & 9,1 \\
\hline 2010 & 05 & 22,7 \\
\hline 2011 & 06 & 27,3 \\
\hline 2013 & 02 & 9,1 \\
\hline \multicolumn{3}{|l|}{ Periódico } \\
\hline Revista Cadernos de Saúde Pública & 05 & 22,8 \\
\hline Human Resources for Health & 03 & 13,8 \\
\hline Gaceta Sanitária & 02 & 9,1 \\
\hline Revista Ciência \& Saúde Coletiva & 02 & 9,1 \\
\hline Physis: Revista de Saúde Coletiva & 02 & 9,1 \\
\hline Revista Saúde e Sociedade & 02 & 9,1 \\
\hline Revista Latino-Americana de Enfermagem & 01 & 4,5 \\
\hline Revista Brasileira de Educação Médica & 01 & 4,5 \\
\hline Interface: Comunicação, Saúde e Educação & 01 & 4,5 \\
\hline Revista de Administração Pública & 01 & 4,5 \\
\hline Texto \& Contexto Enfermagem & 01 & 4,5 \\
\hline Outro* & 01 & 4,5 \\
\hline \multicolumn{3}{|l|}{ Local de estudo } \\
\hline Não delimitado & 06 & 27,3 \\
\hline Rio de Janeiro & 05 & 22,8 \\
\hline São Paulo & 04 & 18,1 \\
\hline Minas Gerais & 03 & 13,6 \\
\hline Rio Grande do Sul & 02 & 9,1 \\
\hline Ceará & 01 & 4,5 \\
\hline Santa Catarina & 01 & 4,5 \\
\hline Total & 22 & - \\
\hline
\end{tabular}

Fonte: Os autores.

Nota: *Coletânea de debates e trabalhos inéditos da Conferência Internacional sobre Pesquisas em Recursos Humanos em Saúde, realizada no Rio de Janeiro em junho de 2010. 
No que se refere à distribuição temporal, 50\% dos artigos foram publicados no biênio 2010/2011. Quanto ao local de realização das pesquisas, 12 $(54,5 \%)$ foram desenvolvidas em municípios de grande porte ou estados; oito $(36,5 \%)$ não especificaram os locais de estudo; uma $(4,5 \%)$ estudou municípios de pequeno porte (MPPs) e uma (4,5\%) teve como base 52 municípios, sendo $85 \%$ destes MPPs.

Ao analisar a localização das instituições às quais os autores estavam vinculados no momento da publicação, verificou-se que as situadas no estado do Rio de Janeiro ( $\mathrm{N}=7$ ) foram as que mais publicaram, seguindo-se os estados de Minas Gerais e São Paulo, com quatro cada um. Instituições internacionais estavam presentes em dois artigos. As instituições da região Sudeste foram as que mais apresentaram artigos (15), podendo-se inferir a relação das publicações com a distribuição de cursos de pós-graduação, pois a maioria dos programas de mestrado e doutorado encontra-se nas grandes cidades do Sudeste e do Sul do Brasil (Brasil, 2010).

Quanto ao tipo de instituição, quase a totalidade dos artigos $(\mathrm{N}=21)$ era de autores vinculados a instituições de ensino, como docente ou discente, e em um artigo o autor era vinculado a um órgão internacional, no caso a Organização Mundial da Saúde (OMS). Em quatro artigos, houve parceria de instituições de ensino com serviços, o que sugeriu uma possível integração ensino-serviço.

Com base na concepção de gestão do trabalho de Ramos e colaboradores (2009) e da leitura dos artigos selecionados, optou-se por construir duas categorias temáticas: as concepções de gestão de trabalho e as questões relacionadas à vida funcional do trabalhador, como ingresso, provimento, movimentação e garantia de direitos trabalhistas, devido ao fato de estarem presentes em todos os estudos analisados.

\section{Concepções de gestão do trabalho}

Dos 22 artigos estudados, em nove se discutiram conceitos de gestão do trabalho. Destes, dois artigos publicados em 2002 indicaram que a gestão do trabalho havia deixado de ser considerada gestão de pessoal próprio e que os gestores públicos desempenhavam cada vez menos a função de empregadores diretos e atuavam mais como instrumentos de contratação de empresas privadas e cooperativas (Dal Poz, 2002), com ampliação da visão instrumental e pragmática das questões de recursos humanos (RH), deixando de se considerar a humanização dos que trabalhavam em saúde (Machado e Pereira, 2002). Artigo publicado em 2008 amplia essa análise e refere que, apesar das inovações nos processos de gestão e das renomeações dos termos, não houve avanço expressivo com relação à autonomia dos gestores de RH, com prevalência do cumprimento de funções burocráticas (Pierantoni et al., 2008). 
Tal situação pode ser ainda mais marcante nas organizações hospitalares, pois Morici e Barbosa (2013), ao analisarem a gestão de RH em hospitais, apontam que as ações prioritárias nessa área são a gestão da entrada e da saída dos profissionais e a implementação de alavancas para melhoria de desempenho dos trabalhadores.

Outros cinco artigos, datados do biênio 2010-2011, já apresentavam o que consideraram como uma evolução do conceito de gestão do trabalho, não mais restrita ao campo de ' $\mathrm{RH}^{\prime}$ como insumos, expandindo-se para um modelo conceitual que considerava a gestão do trabalho e educação na saúde com ênfase na contratação de trabalhadores, capacitação e formação, avaliação, remuneração, condições de trabalho, regulação das relações sociais de trabalho e gestão do processo de trabalho, envolvendo os diferentes níveis de governo (Pierantoni e Garcia, 201 1; Scalco, Lacerda e Calvo, 2010; Vieira, Amâncio Filho e Oliveira, 2004; Mendonça et al., 2010; Lacaz et al., 2010).

Pierantoni, Varella e França (2004) explicam a evolução do conceito de gestão do trabalho como uma superação do termo ' $\mathrm{RH}$ ', este oriundo da ciência da administração (igualado nos mesmos níveis de recursos financeiros e materiais), pela incorporação do conceito de FTS - termo marxista, que vem das ciências políticas e considera fenômenos do mercado de trabalho e da sociologia do trabalho, englobando o trabalho, o trabalhador e suas relações.

Verificou-se convergência das concepções discutidas nestes últimos artigos com a definição de Ramos e colaboradores (2009), como as questões relacionadas à vida funcional do trabalhador e as condições de trabalho mas não houve menção da participação dos trabalhadores na formulação de políticas e na criação de espaços de negociação.

O Ministério da Saúde (MS), ao criar em 2003 a Secretaria de Gestão do Trabalho e da Educação na Saúde (SGTES), apresentou avanços nessa concepção, pois ao formular o conceito de gestão do trabalho destacou o papel do trabalhador como sujeito e agente transformador de seu ambiente e não apenas um mero recurso humano. Pressupunha como requisitos básicos, dentre os já mencionados, processos de formação, desenvolvimento profissional e educação permanente dos trabalhadores (Brasil, 2009).

Além disso, seis artigos fizeram menção sobre a importância da SGTES para a área de gestão do trabalho, por sua atuação em projetos de cooperação técnica com municípios e estados, na formulação de políticas orientadoras da gestão, formação, qualificação e regulação dos trabalhadores; no desencadeamento de iniciativas, como a Política Nacional de Educação Permanente em Saúde, o Pró-Saúde, a Mesa Nacional de Negociação Permanente no SUS (MNNP/SUS), as diretrizes para a elaboração do PCCS/SUS e a norma operacional básica/NOB-RH-SUS, e o Programa de Desprecarização do Trabalho no SUS (DesprecarizaSUS) (Buchan, Fronteira e Dussault, 2011; 
Pierantoni e Garcia, 2011; Scalco, Lacerda e Calvo, 2010; Mendonça et al., 2010; Junqueira et al., 2009). Portanto, houve referências de associações de mudanças induzidas pela SGTES, especialmente em secretarias estaduais de saúde e nas secretarias de capitais, mas com a ressalva de que ainda faltam demonstrações de capacidade de acompanhamento e avaliação dessas políticas e dos instrumentos utilizados pelos gestores para operacionalizá-las (Pierantoni et al., 2008).

\section{Provimento e garantia de direitos trabalhistas}

Conforme já comentado, todos os artigos analisados apresentaram questões referentes ao ingresso, provimento, movimentação e direitos trabalhistas e suas condições referentes à expansão dos empregos públicos na esfera municipal. A Reforma Sanitária Brasileira, o princípio da descentralização e da municipalização desencadearam alterações na organização, no funcionamento e na gestão dos serviços, principalmente no que se refere à atenção básica à saúde (ABS), com adesão dos municípios à Estratégia Saúde da Família (ESF). Consequentemente, a FTS tornou-se essencialmente vinculada aos municípios, e os empregos da esfera federal perderam importância no mercado de trabalho ao longo dos anos (Machado e Pereira, 2002).

Segundo Buchan, Fronteira e Dussault (2011), no Brasil, em 2005, havia 715.137 médicos, enfermeiros e dentistas que trabalhavam em serviços de saúde, dos quais $52 \%$ estavam empregados no setor público; destes, dois terços $(68 \%)$ ) atuavam vinculados em municípios (o que representou um aumento de $22,8 \%$ para médicos, $35,8 \%$ para enfermeiros e $100,7 \%$ para os dentistas, em comparação com dados de 1999). Do total de profissionais, $52 \%$ estavam empregados no setor público, e destes, $68 \%$ atuavam vinculados em municípios. Além disso, anteriormente, o mercado de saúde era polarizado por médicos e auxiliares de enfermagem, que absorviam, juntos, $56,0 \%$ dos empregos. Mesmo com esse crescimento significativo, verificaram-se desigualdades entre as categorias e entre as regiões do Brasil (Buchan, Fronteira e Dussault, 2011; Machado e Pereira, 2002).

Com a expansão dos empregos públicos e da capacidade instalada na esfera municipal, foram alteradas tanto as condições de trabalho das equipes como as definições dos papéis e dos modelos de gestão de RH (Machado, Oliveira e Moysés, 2011; Pierantoni et al., 2008). Ressalta-se que, em 1992, os municípios representavam $41,6 \%$ do total de empregos públicos e, em 2005, elevaram sua participação para 68,8\% (Machado, Oliveira e Moysés, 2011). No entanto, isso não resultou automaticamente na transferência de capacidade de gerenciamento para o nível municipal, pois alguns estudos demonstraram que os municípios tornaram-se um dos responsáveis pelo descumprimento das leis trabalhistas estabelecidas pela Constituição Federal 
(Dal Poz, 2002; Scalco, Lacerda e Calvo, 2010; Pierantoni e Garcia, 2011; Silveira et al., 2010; Coelho, Assunção e Belisário, 2009; Junqueira et al., 2009, 2010).

O Quadro 1 apresenta uma síntese das diferentes formas de provimento da FTS, encontradas em 14 dos 22 artigos analisados. Os dados referem-se a resultados de pesquisas realizadas pelos autores (fontes primárias ou secundárias) ou análises de pesquisas já desenvolvidas que tratavam de formas de provimento da FTS.

\section{Quadro 1}

Síntese dos principais dados sobre formas de provimento da FTS - pesquisa realizada nas bases de dados SciELO e PubMed, 2014

Ano de publicação, autores e título Dimensionamento do estudo

2001.

Dal Poz, Mario Roberto.

As políticas e a gestão de $\mathrm{RH}$ em

saúde: 1984-1995.

2002.

Dal Poz, Mario Roberto.

Cambios en la contratación de

recursos humanos: el caso del Programa

de Salud de la Familia en Brasil.

2002.

Machado, Maria Helena; Pereira, Sonia Los recursos humanos y el sistema de salud en Brasil.
Pesquisa realizada em 1996

nos municípios de Niterói e

Angra dos Reis (RJ).

Apresenta dados sobre as

formas de contratação de

diferentes categorias do PSF e

em diferentes regiões do país.

médicos e enfermeiros do PSF

de caráter nacional.
Formas de provimento da FTS

Segundo o texto, foi previsto o enquadramento de todos os

funcionários no regime estatutário em Niterói, com a criação da

Fundação Municipal de Saúde, enquanto na Secretaria Municipal de

Saúde de Angra dos Reis prevalecia o regime celetista.

No artigo, o autor comenta resultados de investigações realizadas por:

- Motta (1999), no estado do Mato Grosso, em que 79,2\% dos

trabalhadores do PSF foram contratados por prestação de serviços.

- Machado (1999), em pesquisa de âmbito nacional, com profissionais

médicos e enfermeiros em que verificou que os contratos temporários eram a modalidade mais frequente, com exceção para a região Sul, onde predominaram os empregos públicos.

- Solla (1999), em pesquisa realizada em 997 municípios brasileiros, constatou que $49 \%$ dos médicos e $44 \%$ dos enfermeiros tinham contratos temporários; os governos municipais eram responsáveis por $84,9 \%$ dos vínculos empregatícios e as cooperativas, por 4,7\%.

As autoras citam uma investigação realizada por Machado e colaboradores (2000), que revelou que $45,8 \%$ dos médicos e $43,7 \%$ dos enfermeiros ingressaram por meio de contratos temporários e que havia ausência de padronização de formas de contratação entre as regiões; na região Sul, $41,7 \%$ dos médicos e $44,7 \%$ dos enfermeiros foram contratados por estatuto; na Centro-Oeste, $50 \%$ dos médicos e enfermeiros foram contratados pela Consolidação das Leis do Trabalho (CLT); e na região Norte, $67 \%$ dos médicos e $53 \%$ dos enfermeiros foram contratados por meio de contratos temporários. 


\section{Continuação - Quadro 1}

Síntese dos principais dados sobre formas de provimento da FTS - pesquisa realizada nas bases de dados SciELO e PubMed, 2014

Ano de publicação, autores e título Dimensionamento do estudo

Formas de provimento da FTS

2004.

Vieira, Ana Luiza Stiebler; Amâncio Filho,

Antenor; Oliveira, Eliane dos Santos.

Mercado de trabalho em saúde na

região Sudeste-Brasil: a inserção da

equipe de enfermagem.

2007.

Jorge, Maria Salete Bessa et al.

Gestão de recursos humanos

nos centros de atenção psicossocial

no contexto da Política de

Desprecarização do Trabalho no

Sistema Único de Saúde.

2008.

Tomasi, Elaine et al.

Perfil sociodemográfico e

epidemiológico dos trabalhadores

da atenção básica à saúde nas

regiões Sul e Nordeste do Brasil.

2009.

Coelho, Maria Cristina Ramos de Vasconcelos; Assunção, Ada Ávila; Belisário, Soraya Almeida.

Employment and sociodemographic characteristics: a study of increasing precarity in the health districts of Belo Horizonte, Brazil.

\section{Análise das informações}

referentes às categorias da

equipe de enfermagem na

região Sudeste da pesquisa

Assistência Médico-Sanitária

(AMS/1999), do Instituto

Brasileiro de Geografia e

Estatística.

Pesquisa realizada com

trabalhadores de três centros

de atenção psicossocial de

Fortaleza (CE) no período de

2005 a 2006

Pesquisa realizada no ano de

2005 em 41 municípios com

mais de cem mil habitantes das

regiões Sul e Nordeste.

Estudo realizado na Secretaria

Municipal de Saúde de Belo

Horizonte, com dados do

período de 2002 a 2006
No final dos anos 1990, o vínculo próprio prevalecia em todas as categorias da equipe de enfermagem (91,1\%). Entretanto, 11,3\% da força de trabalho dos enfermeiros, 8,6\% dos técnicos, 9,2\% dos auxiliares e 5,7\% dos atendentes estavam vinculados sob formas flexibilizadas.

Constataram-se diversos tipos de vínculos empregatícios (CLT, estatutários, terceirizados, cooperativas e outros) para todas as categorias profissionais. Nesses serviços, havia flexibilização das relações trabalhistas, caracterizada pela terceirização, pela contratação direta de forma temporária ou por meio de cooperativas, bolsas de trabalho e estágios remunerados.

Comparação entre equipes da ESF e do modelo tradicional da ABS. O ingresso por concurso público alcançou pouco mais de $40 \%$ dos trabalhadores, sendo maior no modelo de atenção tradicional do que na ESF nas duas regiões. O vínculo de trabalho tipicamente precário alcançou aproximadamente 38\% dos trabalhadores da ABS (Nordeste, 42\%; Sul, 30\%).

Análise das diferenças entre os trabalhadores temporários e permanentes (estáveis). Verificou-se um crescimento de 305,0\% no número total de trabalhadores temporários e 9,36\% no número total de trabalhadores permanentes no período. A proporção era de 5,49 trabalhadores permanentes para cada trabalhador temporário em 2002. Em 2006, a proporção mudou para 1,48 trabalhador permanente para cada trabalhador temporário. Embora tenha havido um aumento global de 55,03\% no número total de trabalhadores, a relação entre os trabalhadores com contratos temporários e permanentes mudou ao longo do período, indicando uma tendência de maior crescimento na categoria temporário em comparação com a de permanentes. 
Continuação - Quadro 1

Síntese dos principais dados sobre formas de provimento da FTS - pesquisa realizada nas bases de dados SciELO e PubMed, 2014

Ano de publicação, autores e título Dimensionamento do estudo

2010.

Scalco, Sirlesia Vigarini; Lacerda,

Josimari Telino; Calvo, Maria Cristina

Marino.

Modelo para avaliação da gestão de

recursos humanos em saúde.

2010.

Junqueira, Tulio da Silva et al.

As relações laborais no âmbito da

municipalização da gestão em saúde

e os dilemas da relação

expansão/precarização do trabalho

no contexto do SUS.

2010.

Silveira, Denise Silva et al.

Gestão do trabalho, da educação, da

informação e comunicação na

atenção básica à saúde de

municípios das regiões Sul e

Nordeste do Brasil.

2010.

Lacaz, Francisco Antonio de Castro et al.

Qualidade de vida, gestão do

trabalho e plano de carreira como

tecnologista em saúde na atenção

básica do SUS em São Paulo, Brasil.
Resultados da pesquisa

Pesquisa realizada em 15 dos

22 municípios da macrorregião

de Florianópolis (SC) no

período de 2006 a 2007,com

diversas categorias.

Estudo desenvolvido em 2007,

macrorregião sanitária Leste

Sul do estado de Minas Gerais.

Pesquisa realizada no ano de

2005 em 41 municípios com

mais de cem mil habitantes das

regiões Sul e Nordeste.

denominada Avaliação da gestão do trabalho como

tecnologia em saúde na ABS do

SUS no estado de São Paulo,

realizada no período de 2006

a 2008 . com equipes da ESF, na

\section{Formas de provimento da FTS}

Verificou que $47 \%$ dos municípios estudados possuíam mais de $50 \%$ de trabalhadores estatutários. Apenas um dos 15 municípios tinha pelo menos $94,0 \%$ de seus trabalhadores contratados em Regime Jurídico Único.

Os resultados apontaram que 93,3\% das prefeituras respondiam diretamente pelas contratações dos profissionais. Alguns municípios utilizavam mais de uma forma de vinculação. As contratações temporárias e de prestação de serviços predominavam amplamente sobre todas as outras formas. Para profissionais de nível superior e agentes comunitários de saúde (ACSs), os contratos temporários por prestação de serviços foram citados por 75,6\% dos secretários municipais de saúde como a forma de vinculação utilizada, e 24,4\% citaram tipos de contratos que garantiam proteção social (13,3\% estatutários e 11,1\% CLT).

Este trabalho faz parte da mesma pesquisa do artigo de Tomasi e colaboradores (2008), já citado. A maior prevalência de vínculo precário de trabalho foi encontrada entre os ACSs (53\%). O contrato temporário foi o tipo de vínculo mais frequente (Sul=49\% e Nordeste $=67 \%$ ). Em relação à forma de ingresso, $40 \%$ dos trabalhadores de ambas as regiões ingressaram por meio de concurso público. O ingresso por concurso foi menor para ACSs (23\%), trabalhadores de nível elementar (29\%) e outros profissionais de nível médio (39\%).

O vínculo de trabalho dos profissionais contemplados pelos PCCSs era por estatuto em São Paulo e Campinas e por CLT em Guarulhos. Previam o concurso público como única forma de ingresso na carreira nos municípios em estudo. 
Continuação - Quadro 1

Síntese dos principais dados sobre formas de provimento da FTS - pesquisa realizada nas bases de dados SciELO e PubMed, 2014

Ano de publicação, autores e título Dimensionamento do estudo

2011.

Marsiglia, Regina Maria Giffoni.

Perfil dos trabalhadores da atenção

básica em saúde no município de

São Paulo: região norte e central da

cidade.

2011.

Santos, Karina Tonini et al.

Agente comunitário de saúde: perfil adequado à realidade do PSF?

2013.

Morici, Marina Campos;

Barbosa, Allan Claudius Queiroz.

A gestão de RH em hospitais do SUS

e sua relação ao modelo de

assistência: um estudo em hospitais

de Belo Horizonte, MG.
Pesquisa realizada com

trabalhadores que atuavam na

ABS na cidade de São Paulo

(SP) em 2008-2009.

(1)

\section{Formas de provimento da FTS}

Pesquisaram-se trabalhadores que atuavam em unidades básicas de

saúde (UBSs) organizadas exclusivamente na forma de programas em saúde ou da ESF, bem como das unidades mistas, onde coexistiam os dois modelos de atenção. Quase todos os entrevistados que trabalhavam nas UBSs organizadas conforme o modelo de programas de saúde, isto é, 92,3\%, eram funcionários públicos estatutários e efetivos, e 4,7\% eram regidos pela CLT; 1,0\% deles eram estatutários e recebiam complementação salarial pelas instituições parceiras, 0,3\% eram por CLT por meio das instituições parceiras e 1,6\% eram outros casos. Entre os entrevistados que trabalhavam nas UBSs com equipes da ESF implantadas, a situação foi inversa: 77,0\% apresentavam vínculo exclusivo pela CLT, contratados pelas instituições parceiras; $14,8 \%$ eram funcionários públicos concursados; $6,6 \%$ apresentavam duplo vínculo (estatutário + CLT), recebendo complementação salarial por meio de instituições parceiras, e 1,2\% deles eram funcionários públicos celetistas.

Estudo realizado nos anos Ocorrência de diversas modalidades de contratação, com vínculos que 2000, com os profissionais ACSs em quatro municípios de pequeno porte do noroeste se distribuíam desde os mais formais e protegidos pela legislação vigente até os chamados contratos 'informais', com a existência de contratos de caráter precário, muitos sem garantias trabalhistas. paulista.

Pesquisa (qualitativa) com

Pesquisou dois hospitais públicos e dois privados. Os hospitais gestores e profissionais de $\mathrm{RH}$ e análise de documentos em hospitais de Belo Horizonte (MG) em 2011.

públicos vinculavam seus funcionários por meio de estatuto, e os privados, por meio de pessoas jurídicas ou residentes para os médicos, e os demais trabalhadores segundo as regras da CLT. Os profissionais médicos, tanto nos hospitais públicos como nos privados, eram selecionados por mecanismos informais.

Nos hospitais públicos, o processo de recrutamento e seleção era realizado por meio de dois processos seletivos paralelos: concurso público e processo seletivo simplificado. Nos hospitais privados, o processo seletivo era realizado internamente, e a contratação era segundo as regras da CLT.

Fonte: Os autores.

Nota: PSF: Programa Saúde da Família; ESF: Estratégia Saúde da Família; ABS: Atenção Básica à Saúde; ACS: Agente comunitário de saúde. PCCS: Plano de carreira, cargos e salários. 
Os resultados e discussões contidos nos artigos apresentaram a diversidade de formas de admissão adotadas nos municípios/instituições, caracterizando o que ficou conhecido como flexibilização e precarização das relações de trabalho. Flexibilização que se traduziu na multiplicidade de formas e mecanismos de terceirização dos serviços por meio de empresas privadas ou cooperativas, em que o gestor público era cada vez menos um empregador direto; ou na contratação direta, mas por meio de contratos de prestação de serviços por prazo determinado, estágios, cargos comissionados etc., propiciando a precarização dos vínculos e das relações de trabalho (Dal Poz, 2002; Buchan, Fronteira e Dussault, 2011; Coelho, Assunção e Belisário, 2009; Carneiro Jr., Nascimento e Costa, 2013). Vínculos precários foram citados como aqueles que não ofereciam benefícios assistenciais (aposentadoria, licença médica) ou outros benefícios relacionados à legislação trabalhista, como férias, $13^{\circ}$ salário, horas extras, estabilidade etc. (Dal Poz, 2002; Buchan, Fronteira e Dussault, 2011; Junqueira et al., 2010; Coelho, Assunção e Belisário, 2009).

Para o termo trabalho precário, há diferentes concepções: enquanto para os gestores do SUS está relacionado aos vínculos de trabalho, diretos ou indiretos, mas que não garantem direitos trabalhistas e previdenciários consagrados em lei, para entidades sindicais que representam os trabalhadores caracteriza-se não apenas como ausência de direitos trabalhistas e previ-denciários, mas também como ausência de concurso público ou processo seletivo público para cargo permanente ou emprego público (Brasil, 2006a).

Notou-se, portanto, que a expansão dos empregos públicos pela implantação do Programa de Agentes Comunitários de Saúde e da ESF, em um primeiro momento, não resultou em aumento de oportunidades de trabalhos formais, especialmente os que ofereciam estabilidade e garantias trabalhistas, pois desde o início foi marcada por estratégias de flexibilização das relações de trabalho (Machado, Oliveira e Moysés, 2011), uma vez que se recorria a formas alternativas de contratação de pessoal - como organizações não governamentais, cooperativas, associações de moradores, fundações, associações religiosas, organizações da sociedade civil de interesse público e organizações sociais, entre outras -, considerando-se que o aumento do efetivo de trabalhadores na esfera pública se mostrou incompatível com as premissas de controle do déficit público em face das exigências macroeconômicas (Carneiro Jr., Nascimento e Costa, 2013; Santos et al., 2011; Pierantoni et al., 2008).

Alguns gestores municipais defendiam a ideia de que formas de contratos precários permitiam oferecer salários mais atrativos e, portanto, selecionar profissionais com um perfil mais adequado para as atividades da ABS e que seriam uma alternativa ante a necessidade mais imediata de complementação do quadro de pessoal até a realização de concursos públicos (Silveira 
et al., 2010). Outros se preocupavam com a realização de concursos públicos para substituição dos quadros terceirizados em todas as categorias profissionais, o que possibilitaria vínculos mais estáveis, com impacto positivo na fixação de profissionais na ESF (Mendonça et al., 2010).

Estudo verificou que nas instituições hospitalares públicas a falta de provisão de profissionais aprovados em concurso tornou necessária a contratação de trabalhadores por meio de vínculos tidos como flexíveis, garantindo assim o número adequado de profissionais para o atendimento assistencial. Essa contratação contrariava as leis vigentes, mas foi vista como única alternativa para o pleno funcionamento das instituições de saúde pesquisadas. Concluiu-se neste caso que a tentativa de garantir o ingresso de profissionais mediante a realização de concursos públicos gerou uma situação paralela de contratação irregular (Morici e Barbosa, 2013).

Com o intuito de enfrentar o problema da existência de vínculos de trabalho precários, a SGTES/MS criou o DesprecarizaSUS (Junqueira et al., 2009). No entanto, estudo com gestores de $\mathrm{RH}$ de secretarias municipais e estaduais de saúde apontou que 32,4\% deles desconheciam o DesprecarizaSUS e 40,1\% o conheciam, mas dele não participavam; e que, independentemente desse programa, os gestores de RH declararam empreender ações para resolução de vínculos em situação precária, e a realização de concursos e processos seletivos públicos foram as ações mais citadas, o que sinalizou a não capilaridade do DesprecarizaSUS (Pierantoni et al., 2008). Outros trabalhos fizeram uma correlação positiva, mencionando como reflexo da política de 'desprecarização do trabalho' da SGTES/MS a predominância de vínculos estatutários entre os profissionais médicos e enfermeiros das equipes da ESF dos municípios de Aracaju (SE), Belo Horizonte (MG), Florianópolis (SC) e Vitória (ES) (Mendonça et al., 2010). Já o Comitê Nacional Interinstitucional de Desprecarização do Trabalho e as Mesas de Negociação Permanente do SUS foram citados como fatores de contribuição para a revisão dos processos de terceirização e precarização do trabalho na saúde no Brasil (Machado, Oliveira e Moysés, 2011).

Outros resultados apontaram declínio da prática de contratação do trabalho precário na ESF no período de 2001 a 2009, em que a maioria dos municípios brasileiros realizou contratação direta para todas as ocupações de saúde, alcançando valores superiores a 90\% (Machado, Oliveira e Moysés, 2011 ). A contratação direta de ACS foi a que registrou maior aumento (de $82,4 \%$ para $95,5 \%$ ), o que atenuou os efeitos da terceirização e do trabalho precário nessa categoria. Também a prática da terceirização da FTS na ESF diminuiu de forma vigorosa na década de 2000, atingindo 6,9\% para a categoria de médicos e 4\% para a de odontólogos (Girardi et al., apud Machado, Oliveira e Moysés, 2011). Resultados de pesquisa realizada na ABS em MPP no norte do Paraná, em 2010, apontou que 77,2\% dos profissionais foram 
admitidos por meio de vínculos formais (estatutário ou celetista), mas para a categoria médica este percentual era de 50,6\% (Nunes, Carvalho e Santini, 2012).

Quanto às dificuldades de atração e fixação de profissionais na ABS, a categoria médica foi a mais mencionada, consistindo este um dos grandes desafios, principalmente em áreas remotas, zona rural ou em MPP (Buchan, Fronteira e Dussault, 201 1; Scalco, Lacerda e Calvo, 2010; Junqueira et al., 2010; Mendonça et al., 2010; Lacaz et al., 2010; Machado, Oliveira e Moysés, 2011). A oferta de vagas em outros municípios, muitas vezes com melhores salários, e a busca por melhores condições de trabalho e de vida podem fazer com que ocorra uma rotatividade acentuada dos profissionais. Conforme Junqueira e colaboradores (2010), 53,3\% dos secretários afirmaram haver rotatividade em seus municípios, e os médicos foram citados como os profissionais que apresentavam maior rotatividade $(83,3 \%)$, seguidos pelos enfermeiros $(29,2 \%)$.

Segundo gestores municipais e federal, faltam médicos no país e existem importantes vazios assistenciais decorrentes dessa escassez (Carvalho e Souza, 2013). No entanto, setores da medicina, como as corporações médicas, defendem a ideia de que o problema não é de escassez de médicos, e sim de má distribuição e pouca atratividade do setor público, principalmente no que se refere às condições de trabalho e remuneração. Acreditam que, com o aumento de vagas em cursos de medicina, a quantidade de médicos seria suficiente para atender à demanda, e defendem também a necessidade de uma carreira nacional para os médicos no SUS, semelhante às carreiras do Poder Judiciário. No entanto, o projeto de lei n. 7.331/2014, que tinha como objetivo a criação de uma carreira nacional para os médicos do SUS, por meio de alteração da lei n. 12.871, de 22/10/13, que instituiu o Programa Mais Médicos, foi arquivado na Câmara dos Deputados em setembro do mesmo ano, por contrariar o disposto no artigo 61, § 1 , inciso II, alínea "a", da Constituição Federal, que preconiza que são de iniciativa privativa do presidente da República as leis que criem cargos, funções ou empregos públicos na administração direta e autárquica ou aumento de sua remuneração (Brasil, 2014).

As dificuldades de provimento e fixação do profissional médico já eram sentidas desde o início da ESF, pois há registros de que 22 municípios dos estados do Tocantins, Acre e Maranhão possuíam em seus quadros médicos estrangeiros em 1999, sendo a maioria de Cuba (Dal Poz, 2002). Outro artigo evidenciou que mais de $70 \%$ dos profissionais de saúde se encontravam nas regiões Sul e Sudeste, vários municípios das regiões Norte e Nordeste não dispunham de nenhum médico e havia concentração de profissionais médi$\cos (65 \%)$ nos centros urbanos, em especial nos mais desenvolvidos econômica e socialmente, demonstrado pela proporção de 3,28 médicos/1.000 habitantes para as capitais e 0,53 médico/1.000 habitantes para o interior 
(Machado e Pereira, 2002). Em Nunes e colaboradores (2015), na ABS dos MPPs da macrorregião norte do estado do Paraná, a proporção encontrada foi de 0,51 médico/1.000 habitantes. Essas dificuldades continuam repercutindo no acesso e na qualidade dos serviços prestados, o que tem gerado insatisfação por parte dos usuários em diferentes localidades (Carvalho e Souza, 2013).

O desafio de fixar o profissional médico tem sido enfrentado pelos gestores de diferentes formas: equiparação do salário do médico da ABS com o dos médicos dos serviços secundários; estabelecimento de incentivos/complementação salarial para os profissionais da ESF conforme critérios de risco e vulnerabilidade dos locais de trabalho; regularização de vínculos ou disponibilização de vínculos adicionais, como a realização de plantões; desenvolvimento de estratégias de qualificação; diversificação de ações assistenciais e melhoria na articulação entre os serviços (Carvalho e Souza, 2013). No decorrer dos anos, verificaram-se resultados exitosos como o percentual significativo de permanência dos profissionais ( $40 \%$ a $60 \%$ dos médicos) nos municípios de Aracaju, Belo Horizonte e Vitória em 2008 (Mendonça et al., 2010). Além disso, o MS, em parceria com o Ministério da Educação, lançou o Programa de Valorização do Profissional de Atenção Básica (Provab), com a finalidade de estimular e valorizar os profissionais de saúde que atuam na ABS e levá-los para áreas de maior carência (Brasil, 2011). E no ano de 2013 instituiu-se, por meio da lei n. 12.871, o Programa Mais Médicos, com ações voltadas para a reordenação das ofertas de cursos e vagas em cursos de medicina; o estabelecimento de novos parâmetros para a formação médica e o aperfeiçoamento de médicos na área da ABS, mediante integração ensino-serviço, inclusive com a possibilidade de intercâmbio internacional (Brasil, 2013).

A precarização dos vínculos e a falta de incentivos para os profissionais da área não estimulam a permanência dos trabalhadores nas funções, levando-os a buscar melhores oportunidades e a encararem os serviços como eventuais ou temporários (Scalco, Lacerda e Calvo, 2010; Machado e Pereira, 2002; Coelho, Assunção e Belisário, 2009; Morici e Barbosa, 2013). Também podem deixar os profissionais à mercê da instabilidade político-partidária e das diferenças entre governos que se sucedem no poder, desestabilizando, dessa forma, o valor transformador do SUS (Junqueira et al., 2009). Em geral, trabalhadores admitidos por formas precárias têm menor segurança no emprego e no controle sobre suas horas de trabalho, piores perspectivas de carreira, acesso limitado à educação e formação, redução de oportunidades e salários mais baixos (Coelho, Assunção e Belisário, 2009; Jorge et al., 2007).

Estudo que analisou a rotatividade de profissionais de saúde em 15 municípios da macrorregião da Grande Florianópolis (SC) indicou altas taxas de rotatividade em 50,0\% deles e taxas de rotatividade aceitáveis em outros 47,0\% (Scalco, Lacerda e Calvo, 2010). Pela variação dos percentuais, verificou-se 
que não havia uniformidade de condições e que a rotatividade ainda era um desafio a ser vencido para metade dos municípios dessa macrorregião.

Pesquisa realizada na ABS da cidade de São Paulo, em 2009, encontrou os seguintes resultados: $23,7 \%$ dos profissionais trabalhavam na UBS há apenas um ou dois anos, 14,2\% há três ou quatro anos e 20,9\% há cinco ou seis anos, o que indicou um processo de renovação e incorporação de novos trabalhadores (Marsiglia, 2011). Quanto à rotatividade de profissionais ACSs, pesquisa realizada em quatro MPPs do noroeste paulista e publicada em 2011 identificou que, dos ACSs entrevistados, 47,4\% atuavam há menos de um ano no PSF, caracterizando a alta rotatividade desses profissionais (Santos et al., 2011). Em Nunes e colaboradores (2015), os ACSs eram os profissionais que estavam atuando há menos tempo nas equipes da ESF, pois $65,8 \%$ se encontravam de um a seis anos nos serviços.

Os fatores que dificultam a fixação dos profissionais são: remuneração e condições de trabalho aquém das expectativas, inclusive com dificuldade de acesso aos locais de trabalho; exigências de cumprimento de carga horária integral ou de dedicação exclusiva; baixa oportunidade de progressão profissional e sem vinculação a um PCCS; ausência de formação prévia compatível como o modelo da ESF; falta de profissionais; vínculos precários e sem seleção pública; e falta de integração entre os gestores municipais de uma mesma região (Junqueira et al., 2010; Silveira et al., 2010; Vieira, Amâncio Filho e Oliveira, 2004; Mendonça et al., 2010).

Mesmo em instituições hospitalares, a fixação de profissionais mostrou-se um desafio, principalmente pelo grande impacto causado pela perda de trabalhadores experientes, treinados e habituados às rotinas. Em hospitais públicos, o entrave seria a incapacidade de reposição de pessoal com a agilidade requerida para o bom funcionamento assistencial. Em hospitais privados, a falta de trabalhadores capacitados e as regras da CLT tornaram os processos burocráticos e sem capacidade de dar resposta às demandas com rapidez (Morici e Barbosa, 2013).

Estudos demonstram que a alta rotatividade dos profissionais de saúde gera um aumento de custos para reposição de pessoal, insatisfação no ambiente de trabalho e dificuldades no atendimento ao usuário, pois o vínculo dos profissionais com o serviço e com a população é uma característica indissociável do trabalho em saúde, e o trabalhador com inserção fixa nos serviços tem melhores garantias trabalhistas e está menos sujeito à precarização do trabalho (Scalco, Lacerda e Calvo, 2010; Junqueira et al., 2010). Há necessidade, portanto, além de prover profissionais, de articular mecanismos para a fixação destes e propor mudanças no modelo de atenção e cuidado à saúde, como também na formação desses trabalhadores (Carvalho e Souza, 2013).

Fator citado como importante para a qualidade de vida no e do trabalho e, consequentemente, para a atração e fixação de profissionais foi o PCCS 
(Lacaz et al., 2010). No entanto, sua implantação no SUS ainda não foi consolidada, o que se considera um fator limitante a ser enfrentado (Lacaz et al., 2010; Mendonça et al., 2010; Buchan, Fronteira e Dussault, 2011).

Segundo Dal Poz (2001), nos municípios de Niterói e Angra dos Reis, em 1996, o PCCS representava apenas formalidades, sem qualquer relação com o modelo assistencial ou com a definição de gestão dos sistemas locais. Uma das alternativas encontradas por Niterói para fugir das limitações do PCCS na época foi a contratação de servidores pelas associações de moradores, com repasse de recursos pela prefeitura. Também se verificaram dificuldades em elaborar e implantar um PCCS no nível municipal, bem como suas limitações como instrumento de política de RH.

Entre os resultados de estudos realizados entre os anos de 2005 e 2008, consta que, em 2005, somente $14 \%$ dos profissionais da $\mathrm{AB}$ da região Sul e $17 \%$ da região Nordeste haviam referido acesso a um PCCS; apenas 40\% dos municípios da macrorregião da Grande Florianópolis possuíam PCCSs implantados em 2006; e o PCCS foi implantado na Secretaria Municipal de Saúde de Belo Horizonte em 2007 (Silveira et al., 2010; Coelho, Assunção e Belisário, 2009; Scalco, Lacerda e Calvo, 2010).

Lacaz e colaboradores (2010), em pesquisa realizada em 2008 no estado de São Paulo, verificaram pequena adesão dos municípios estudados aos princípios definidos pelas diretrizes nacionais do PCCS-SUS, como: PCCS exclusivo para trabalhadores do SUS; democratização das relações de trabalho; princípios de compromisso solidário e avaliação de desempenho como processo pedagógico e critérios de mérito para ascensão profissional, como tempo de serviço e escolarização, entre outros. No entanto, verificou-se nos três municípios estudados que seus PCCSs abarcavam trabalhadores de todos os níveis de escolaridade, previam o concurso público como única forma de ingresso na carreira e adotavam prêmios por produtividade e desempenho. Uma lacuna observada pelos autores é que não era mencionado, tanto nas diretrizes nacionais como nos PCCSs analisados, carreiras para cargos de gerenciamento no sistema de saúde, permanecendo estes estritamente vinculados à indicação do gestor municipal.

Artigo publicado por Pierantoni e colaboradores (2008), sobre estudo aplicado em trabalhadores dos órgãos gestores e executores de ações e serviços de saúde de capitais, de secretarias estaduais de saúde e de municípios com população superior a quinhentos mil habitantes, verificou que $47,8 \%$ não contavam com PCCS, apesar de reconhecerem as vantagens desse instrumento, tanto do ponto de vista gerencial quanto na perspectiva dos trabalhadores. Tal situação pode ser ainda mais contundente em MPPs, pois em estudo realizado em 2010 com profissionais da ABS, verificou-se que 79,5\% deles não estavam incluídos em PCCSs (Nunes et al., 2015).

O único artigo tendo como campo de estudo serviços hospitalares de Belo Horizonte identificou que os hospitais públicos ofereciam aos seus trabalhadores 
planos bem definidos e progressões baseadas em tempo de serviço e avaliações de desempenho, enquanto nos privados não existia definição formal de carreira e os aumentos salariais eram concedidos de forma isolada ou como resultado de negociação sindical (Morici e Barbosa, 2013).

Finalmente, segundo Pierantoni e colaboradores (2008), o PCCS ainda é um dispositivo que apresenta muitas barreiras e fragilidades, mesmo que seja apontado como instrumento facilitador da gestão. E para Lacaz e colaboradores (2010), o PCCS tem limitações como instrumento estratégico na gestão do trabalho no SUS e também para a melhoria da qualidade de vida no/do trabalho na $\mathrm{AB}$.

\section{Considerações finais}

O estudo aqui apresentado possibilitou analisar a gestão da força de trabalho no SUS e as estratégias utilizadas pelos municípios para a sua operacionalização ao longo dos anos. Verificou mudança na própria conceituação de gestão de recursos humanos, passando a ser utilizado com maior frequência o termo gestão da força de trabalho, o que pode ter sido potencializado pela criação da SGTES no MS.

Ao término da Década da força de trabalho em saúde: 2006-2015 (Brasil, 2006b), instituída pela OMS para chamar a atenção das autoridades sobre a crise global da FTS, pode-se inferir que houve algumas mudanças importantes, como o processo de expansão dos empregos públicos na esfera municipal e a diminuição de vínculos precários, principalmente na ABS, mesmo com a diversidade de modalidades de contratação ou admissão adotadas pelos municípios.

Embora não haja correlação imediata entre a criação da SGTES e os avanços na diminuição da flexibilização e precarização das relações de trabalho no SUS, não se pode desconsiderar sua importância na indução de políticas de gestão e na inclusão da discussão desses temas nos espaços de gestão e no meio acadêmico. No entanto, dificuldades de atração e fixação de profissionais têm se mantido e ainda são desafios para a gestão, principalmente quanto à categoria médica, o que desencadeou algumas iniciativas por parte do MS, como o Provab e o Programa Mais Médicos - cujos reflexos no mercado de trabalho, na assistência e no processo de formação devem ser acompanhados e estudados, bem como sua consolidação ou não para o provimento de profissionais médicos. Além disso, o PCCS não se efetivou como estratégia para o provimento e a fixação da força de trabalho no SUS.

Este trabalho retratou um ciclo, mas a permanência desses problemas indica a complexidade que caracteriza a gestão da FTS e a necessidade de discussões para seu enfrentamento diante dos novos cenários que se vislum- 
bram e terão importantes repercussões para a gestão no SUS. É determinante para a melhoria da qualidade da atenção à saúde que esses movimentos sejam amplamente discutidos e analisados com trabalhadores, gestores e usuários. Dessa forma, tornam-se necessárias a continuidade e a ampliação de estudos que abordem a temática sobre gestão do trabalho no SUS, como a diversidade de modalidades de contratação, pois se trata de um importante fator que desencadeia conflitos ou tensionamento nas relações entre gestores e trabalhadores, entre trabalhadores e usuários e entre os próprios trabalhadores.

\section{Colaboradores}

Stela Maris Lopes Santini participou da concepção do estudo, coleta, análise dos dados e redação do manuscrito. Elisabete de Fátima Pólo de Almeida Nunes e Brígida Gimenez Carvalho participaram da concepção do estudo, análise dos dados e redação do manuscrito. Francisco Eugênio de Souza contribuiu com a análise dos dados e redação do manuscrito.

Resumen El estudio que dio origen a este artículo presentó los resultados de revisión integrativa de literatura sobre la gestión del trabajo en el Sistema Único de Salud, teniendo como objetivo analizar las diferentes contribuciones científicas en el área, las experiencias y estrategias desarrolladas por los municipios. Se utilizó para la recolección de datos las bases PubMed y SciELO en 2014. Tras la aplicación de los criterios de inclusión y exclusión, se analizaron 22 artículos. El mayor número de éstos se publicó en el bienio 2010-2011 y se refería a municipios de gran porte o estados. Se construyeron dos categorías temáticas: concepciones de gestión de trabajo y cuestiones relacionadas con la vida funcional del trabajador, como aprovisionamiento y garantía de derechos laborales. Se observaron: la evolución del término recursos humanos hacia la concepción de gestión del trabajo; el proceso de expansión de los empleos públicos en la esfera municipal y las diversas formas de selección adoptadas; la desprecarización de los vínculos laborales a partir de los años 2000; las dificultades de atracción y fijación de profesionales y la no consolidación del plan de carrera, cargos y salarios como instrumento estratégico para la gestión del trabajo. Se pusieron de manifiesto la complejidad del tema y la necesidad de constantes estudios por su importancia en el Sistema Único de Salud.

Palabras clave gestión del trabajo; fuerza de trabajo en salud; gestión en salud; recursos humanos en salud; Sistema Único de Salud. 


\title{
Notas
}

\author{
1 Universidade Estadual de Londrina, Programa de Pós-Graduação em Saúde Coletiva, \\ Londrina, Paraná, Brasil. \\ <stelamaris08@gmail.com> \\ Correspondência: Avenida Espírito Santo, n. 353, Jardim Apucarana, CEP 86804-370, \\ Apucarana, Paraná, Brasil. \\ 2 Universidade Estadual de Londrina, Programa de Pós-Graduação em Saúde Coletiva, \\ Londrina, Paraná, Brasil. \\ <alnunes.3@gmail.com> \\ 3 Universidade Estadual de Londrina, Programa de Pós-Graduação em Saúde Coletiva, \\ Londrina, Paraná, Brasil. \\ <brigidagimenez@gmail.com> \\ 4 Universidade Estadual de Londrina, Programa de Pós-Graduação em Saúde Coletiva, \\ Londrina, Paraná, Brasil. \\ $<$ kiko@uel.br>
}

\section{Referências}

BRASIL. Ministério da Saúde. Secretaria de Gestão do Trabalho e da Educação na Saúde. Departamento de Gestão e da Regulação do Trabalho em Saúde. Programa Nacional de Desprecarização do Trabalho no SUS. Brasília: Ministério da Saúde, 2006a. (Série C. Programas, Projetos e Relatórios). Disponível em: <http://bvsms.saude.gov.br/bvs/ publicacoes/cartilhaprogesus.pdf $>$. Acesso em: 11 set. 2015.

BRASIL. Ministério da Saúde. Secretaria de Gestão do Trabalho e da Educação na Saúde. Organização Pan-Americana da Saúde. Reunião regional dos observatórios de recursos humanos em saúde. Chamado à ação de Toronto: 2006-2015 - rumo a uma década de recursos humanos em saúde nas Américas. Brasília: Ministério da Saúde, 2006b. Disponível em: <http://iris.paho.org/xmlui/bitstream/ handle/123456789/3603/Acao-Toronto-20062015.pdf? sequence $=1>$. Acesso em: 3 jan. 2017.
BRASIL. Ministério da Saúde. Secretaria Executiva. Secretaria de Gestão do Trabalho e da Educação na Saúde. Glossário temático: gestão do trabalho e da educação na saúde. Brasília: Ministério da Saúde, 2009. (Série A. Normas e Manuais Técnicos).

BRASIL. Ministério da Educação. Coordenação de Aperfeiçoamento de Pessoal de Nível Superior. Plano Nacional de Pós-Graduação (PNPG) 2011-2020. Documentos setoriais, v. II. Brasília: Capes, 2010. Disponível em: <www. capes.gov.br/images/stories/download/PNPG_ Miolo_V2.pdf>. Acesso em: 8 set. 2015.

BRASIL. Ministério da Saúde. Portaria interministerial n. 2.087, de 1 de setembro de 2011. Institui o Programa de Valorização do Profissional da Atenção Básica. Diário Oficial da União, Brasília, DF, 1 set. 2011. Disponível em: <http://bvsms.saude.gov.br/bvs/ saudelegis/gm/2011/pri2087_01_09_2011. html>. Acesso em: 1 set. 2015. 
BRASIL. Lei n. 12.871, de 22 de outubro de 2013. Institui o Programa Mais Médicos, altera as leis n. 8.745, de 9 de dezembro de 1993, e n. 6.932, de 7 de julho de 1981, e dá outras providências. Presidência da República. Casa Civil. Subchefia para Assuntos Jurídicos. Diário Oficial da União, Brasília, DF, 23 out. 2013. Disponível em: <www. planalto.gov.br/ccivil_03/_ato2011-2014/ 2013/Lei/L12871.htm>. Acesso em: 29 out. 2015.

BRASIL. Projeto de lei n. 7.331, de 1 de abril de 2014. Altera a lei n. 12.871, de 22 de outubro de 2013, que instituiu o Programa Mais Médicos. Câmara dos Deputados. Ficha de tramitação. 2014. Disponível em: <www.camara.gov.br/proposicoesWeb/ fichadetramitacao?idProposicao $=610688 \mathrm{P}$. Acesso em: 3 jan. 2017.

BUCHAN, James; FRONTEIRA, Inês; DUSSAULT, Gilles. Continuity and change in human resources policies for health: lessons from Brazil. Human Resources for Health, London, v. 9, n. 17, p. 1-13, jul. 2011. Disponível em: <www.biomedcentral.com/content/ pdf/1478-4491-9-17.pdf> . Acesso em: 8 jun. 2014.

CAMPOS, Francisco E.; MACHADO, Maria H.; GIRARDI, Sábado N. A fixação de profissionais de saúde em regiões de necessidades. Divulgação em Saúde para Debate, Rio de Janeiro, n. 44, p. 13-24, maio 2009. Disponível em: <www.cnts.org.br/public/arquivos/ Artigo_Campos.pdf>. Acesso em: 13 abr. 2014.

CARNEIRO JÚNIOR, Nivaldo; NASCIMENTO, Vania B.; COSTA, Ieda M. C. Relação entre público e privado na atenção primária à saúde: considerações preliminares. Saúde e Sociedade, São Paulo, v. 20, n. 4, p. 971-979, out.-dez. 2011.

CARVALHO, Mônica S.; SOUZA, Maria F. Como o Brasil tem enfrentado o tema provimento de médicos? Interface: Comunicação, Saúde e Educação, Botucatu, v. 17, n. 47, p. 913-926, nov.-dez. 2013.

COELHO, Maria C. R. V.; ASSUNÇÃO, Ada A.; BELISÁRIO, Soraya A. Employment and sociodemographic characteristics: a study of increasing precarity in the health districts of Belo Horizonte, Brazil. Human Resources for Health, London, v. 7, n. 56, p. 1-13, jul. 2009. Disponível em: <www.humanresources-health.com/content/pdf/14784491-7-56.pdf>. Acesso em: 11 nov. 2015.

DAL POZ, Mario R. As políticas e a gestão de recursos humanos em saúde: 1984 a 1995. Physis: Revista de Saúde Coletiva, Rio de Janeiro, v. 11, n. 2, p. 19-49, jul./dez. 2001.

DAL POZ, Mario R. Cambios en la contratación de recursos humanos: el caso del Programa de Salud de la Familia en Brasil. Gaceta Sanitaria, Barcelona, v. 16, n. 1, p. 82-88, 2002.

JORGE, Maria S. B. et al. Gestão de recursos humanos nos centros de atenção psicossocial no contexto da Política de Desprecarização do Trabalho no Sistema Único de Saúde. Texto \& Contexto Enfermagem, Florianópolis, v. 16, n. 3, p. 417-425, jul.-set. 2007.

JUNQUEIRA, Túlio S. et al. Saúde, democracia e organização do trabalho no contexto do Programa Saúde da Família: desafios estratégicos. Revista Brasileira de Educação Médica, Rio de Janeiro, v. 33, n. 1, p. 122-133, jan.-mar. 2009.

JUNQUEIRA, Túlio S. et al. As relações laborais no âmbito da municipalização da gestão em saúde e os dilemas da relação expansão/precarização no trabalho no contexto do SUS. Cadernos de Saúde Pública, Rio de Janeiro, v. 26, n. 5, p. 918-929, maio 2010.

LACAZ, Francisco A. C. et al. Qualidade de vida, gestão do trabalho e plano de carreira como tecnologista em saúde na atenção básica do Sistema Único de Saúde em São Paulo, Brasil. Cadernos de Saúde Pública, Rio de Janeiro, v. 26, n. 2, p. 253-263, fev. 2010.

MACHADO, Maria H.; OLIVEIRA, Eliane S.; MOYSÉS, Neuza M. N. Tendências do mercado de trabalho em saúde no Brasil. In: PIERANTONI, Célia; DAL POZ, Mario R.; FRANÇA, Tania (orgs.). O trabalho em saúde: abordagens quantitativas e qualitativas. Rio de Janeiro: Cepesc, Uerj, 2011. p. 103-116. 
MACHADO, Maria H.; PEREIRA, Sonia. Los recursos humanos y el sistema de salud en Brasil. Gaceta Sanitaria, Barcelona, v. 16, n. 1, p. 89-93, jan.-fev. 2002.

MARSIGLIA, Regina M. G. Perfil dos trabalhadores da atenção básica em saúde no município de São Paulo: região norte e central da cidade. Saúde e Sociedade, São Paulo, v. 20, n. 4, p. 900-911, out.-dez. 2011.

MENDONÇA, Maria H. M. et al. Desafios para gestão do trabalho a partir de experiências exitosas de expansão da Estratégia Saúde da Família. Ciência \& Saúde Coletiva, Rio de Janeiro, v. 15, n. 5, p. 2.355-2.365, ago. 2010.

MORICI, Marina C.; BARBOSA, Allan C. Q. A gestão de recursos humanos em hospitais do Sistema Único de Saúde (SUS) e sua relação ao modelo de assistência: um estudo em hospitais de Belo Horizonte, Minas Gerais. Revista de Administração Pública, Rio de Janeiro, v. 47, n. 1, p. 205-225, jan./fev. 2013.

NUNES, Elisabete F. P. A.; CARVALHO, Brígida G.; SANTINI, Stela M. L. Gestão do processo de trabalho na rede de atenção básica de saúde em municípios de pequeno porte da região norte do Paraná. Relatório final de pesquisa. Universidade Estadual de Londrina, Londrina, 2012. Digitado.

NUNES, Elisabete F. P. A. et al. Força de trabalho em saúde na atenção básica em municípios de pequeno porte do Paraná. Saúde em Debate, Rio de Janeiro, v. 39, n. 104, p. 29-41, jan.-mar. 2015.

PIERANTONI, Célia R.; GARCIA, Ana C. P. Human resources for health and decentralization policy in the Brazilian health system. Human Resources for Health, London, v. 9, n. 12, p. 1-13, maio 2011. Disponível em: <www.human-resources-health.com/ content/9/1/12>. Acesso em: 8 jun. 2014.
PIERANTONI, Célia R.; VARELLA, Thereza C.; FRANÇA, Tania. Recursos humanos e gestão do trabalho em saúde: da teoria para a prática. In: REGO, Andre F. (org.). Observatório de recursos humanos em saúde no Brasil: estudos e análises. Brasília: Ministério da Saúde, 2004. v. 2. p. 51-70. Disponível em: <www.obsnetims.org.br/uploaded/ 24_1_2014_0_Observatorio_volume_dois. pdf > . Acesso em: 27 maio 2014.

PIERANTONI, Célia R. et al. Gestão do trabalho e da educação na saúde: recursos humanos em duas décadas do SUS. Physis: Revista de Saúde Coletiva, Rio de Janeiro, v. 18, n. 4, p. 685-704, 2008.

RAMOS, Alexandre S. et al. Política de gestão do trabalho e educação permanente na Bahia: o SUS é uma escola. Revista Baiana de Saúde Pública, Salvador, v. 33, n. 1, p. 40-50, jan.-mar. 2009.

SANTOS, Karina T. et al. Agente comunitário de saúde: perfil adequado à realidade do Programa Saúde da Família? Ciência \& Saúde Coletiva, Rio de Janeiro, v. 16, supl. 1, p. 1.023-1.028, 2011.

SCALCO, Sirlesia V.; LACERDA, Josimari T.; CALVO, Maria C. M. Modelo para avaliação da gestão de recursos humanos em saúde. Cadernos de Saúde Pública, Rio de Janeiro, v. 26, n. 3, p. 603-614, maio 2010.

SILVEIRA, Denise S. et al. Gestão do trabalho, da educação, da informação e comunicação na atenção básica à saúde de municípios das regiões Sul e Nordeste do Brasil. Cadernos de Saúde Pública, Rio de Janeiro, v. 26, n. 9, p. 1.714-1.726, set. 2010.

TOMASI, Elaine et al. Perfil sociodemográfico e epidemiológico dos trabalhadores da atenção básica à saúde nas regiões Sul e Nordeste do Brasil. Cadernos de Saúde Pública, Rio de Janeiro, v. 24, supl. 1, p. S193-S201, 2008. 
VIEIRA, Ana L. S.; AMÂNCIO FILHO, Antenor; OLIVEIRA, Eliane S. Mercado de trabalho em saúde na região Sudeste-Brasil: a inserção da equipe de enfermagem. Revista Latino-Americana de Enfermagem, Ribeirão Preto, v. 12, n. 1, p. 134-138, jan.-fev. 2004.

Recebido em 18/10/2015

Aprovado em 06/05/2016 